\title{
An Improvement Approach for Wide-Angle Impedance Matching Using ELC Metasurface Slabs for SIW Slot Array Antennas
}

\author{
Yi Liu $\mathbb{D}^{1}{ }^{1}$ Hu Yang, ${ }^{1}$ Zusheng Jin $\mathbb{D}^{1,2}$ and Jiang Zhu ${ }^{1}$ \\ ${ }^{1}$ College of Electronics Science and Engineering, National University of Defense Technology, Changsha 410073, China \\ ${ }^{2}$ EMC Research and Measurement Center of Navy, Shanghai 200235, China \\ Correspondence should be addressed to Yi Liu; yi_liu@nudt.edu.cn
}

Received 23 February 2018; Revised 14 April 2018; Accepted 23 April 2018; Published 30 April 2018

Academic Editor: Shah Nawaz Burokur

Copyright (C) 2018 Yi Liu et al. This is an open access article distributed under the Creative Commons Attribution License, which permits unrestricted use, distribution, and reproduction in any medium, provided the original work is properly cited.

\begin{abstract}
The effects of mutual coupling in beam scanning arrays increase degradation in gain as the beam is scanned off the broadside. A simple and effective approach for a deployed SIW slot array antenna through the use of artificially structured materials is proposed to improve its performance of wide-angle scanning in the E-plane. Metasurface slabs of one-tenth wavelength electric-inductive-capacitive (ELC) resonators are vertically placed halfway between the adjacent waveguides without changing the antenna structure to realize wide-angle impedance matching (WAIM). The ELC metasurface is designed to operate at a linear region rather than at a resonance region to use its transmission property for greatly weakening mutual couplings. Inserting metasurface slabs between two adjacent waveguides to reduce the mutual coupling can achieve impedance matching at large scan angles. A $10 \times 10$ SIW slot array operating at X-band is modelled and simulated to study how the vertically placed ELC metasurface slabs over the antenna affect the array's beam scanning performance. The simulated results show that the scan range of the antenna is extended from $-50^{\circ}$ to $50^{\circ}$ to $-70^{\circ}$ to $70^{\circ}$ under the criteria that radiating efficiency is greater than $80 \%$.
\end{abstract}

\section{Introduction}

Planar beam scanning antennas play a pivotal role in the applications such as radars and MIMO wireless communication systems. These electronically scanned arrays, like phased arrays, typically suffer from an increasing scan loss as the beam is steered off the broadside [1]. The effect of mutual coupling between the radiating elements is the main cause to mismatch the scan impedance especially at large scan angles, resulting in gain reductions [2]. Placing bulky radomes over the phased arrays is a common approach to overcome the coupling effects [3].

For planar antennas, low-profile structure is an important virtue. In order to retain the low profile, many methods have been presented to improve scanning performance and to eliminate scan blindness. If the limitation is ascribed to the beamwidth of radiating elements, the latest researches proposed several types of wide-beam antenna element to realize wide-angle scanning [4-9]. However, viewing this limitation as an impedance matching problem for conventional antenna elements, an early approach is to locate a thin, high-permittivity dielectric sheet at some distance above a waveguide array [10]. The sheet with well-designed parameters (permittivity, thickness, and height above the array) acts as a shunt susceptance to achieve an impedance match at one scan angle. It is validated to be effective in the $E-, D$ (diagonal), and $H$-planes. Recently, metamaterial sheets of tailored electric and magnetic properties are proposed to increase scanning performance. These metamaterial sheets are parallelly placed over the antenna, resembling dielectric sheets, to improve impedance matching in a wide-angle range $[2,11,12]$. In [11], a type of ELC metamaterial sheet is simulated and validated to improve the scanning performance in the $H$-plane alone. The SSR metamaterial sheet is theoretically studied for an infinite array of dipoles [12] and experimentally validated for a leaky-wave antenna [2] by the same author. But this parallelly placed SSR sheet can only improve the scan range in $H$ - and $D$-planes while the effect is invisible in the E-plane. A metamaterial slab, which is formed by periodically grounded edge-coupled split-ring resonators (PGE-SRRs), is vertically inserted halfway between microstrip elements to obtain low-mutual-coupling performance 


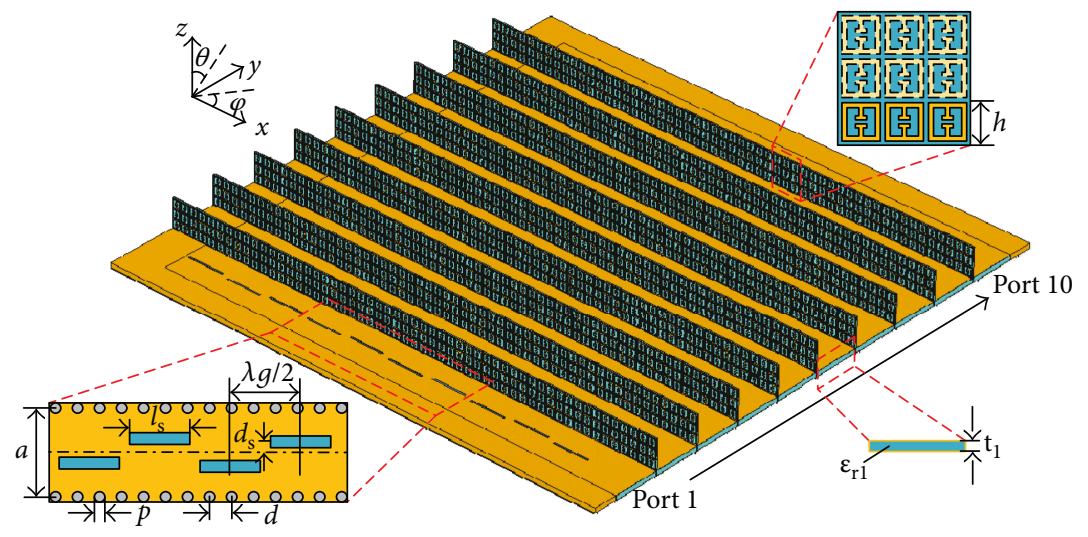

Figure 1: Configuration of the proposed $10 \times 10 \mathrm{SIW}$ slot array antenna covered with an ELC metasurface: $p=0.5 \mathrm{~mm}, d=0.9 \mathrm{~mm}$, and $a=15.0 \mathrm{~mm}$.

[13]. The PGE-SRRs are designed to operate at the resonance region. That is to say, the metamaterial slab can shield the mutual coupling between the elements, but meanwhile it also will block the incident wave at large scanning angles. The paper [13] just gives an example that the scanning range of a microstrip array is extended from $-13^{\circ}$ to $13^{\circ}$ to $-28^{\circ}$ to $28^{\circ}$ after improvement. It cannot be regarded as a wide-angle scanning array.

Waveguide slot arrays are very attractive in microwave antennas for its high gain, high aperture efficiency, and polarization purity. The properties of their radiating slot and the mutual couplings between elements are sensitive to the dielectric slabs or other coverings with different permittivities above the antenna. If covered with dielectric or metamaterial slabs, the antenna must be redesigned because of their effects on the properties of radiating slots [14]. The scanning beams of an SIW slot array are often steered in the E-plane [15], but from [11, 12], we know that the parallelly placed metamaterial sheet over the antenna is effectively invisible for beam steering in the $E$-plane. Based on the considerations above, we aim to improve the range of the scanning angle for a deployed SIW slot array by adding additional metamaterial slabs without changing the antenna's original structure. The introduced ultrathin WAIM slab is composed of subwavelength ELC resonators [16] and a piece of dielectric substrate. They are vertically placed halfway between two adjacent waveguides and used to weaken the mutual coupling phase between two waveguides to improve impedance matching at large scan angles. Thus, we can transform a deployed waveguide slot array into a wideangle scanning array. A $10 \times 10$ SIW slot array is designed to study the proposed approach in the cases without WAIM or with different clapboards. Simulations of the different cases are compared, and the results show that the proposed method is an excellent way to improve the scanning performance at small cost of increasing the antenna's profile and gain loss at small scan angles.

The organization of the paper is as follows. Section 2 describes the overall structure of the SIW slot array with ELC metasurface slabs. In Section 3, the subwavelength ELC resonator is investigated and its property of weakening mutual coupling is discussed. Section 4 gives the qualitative analysis of how the ELC metasurface affects the mutual coupling. The wide-angle scanning performance of the SIW slot array is presented in Section 5. Conclusions are drawn in Section 6.

\section{Description of the Antenna Configuration}

A perspective view of the antenna geometry can be seen in Figure 1 . The $10 \times 10$ SIW slot array is covered by additional metasurface sheets. The slot array is composed of ten waveguides, and each of them has ten radiating slots. The ELC metasurface slabs are located halfway between two adjacent waveguides, standing right on the antenna and align with the metal wall.

The equivalent width $(a)$ of a SIW is related to the diameter of the via $(p=0.5 \mathrm{~mm})$ and the period spacing between two vias $(d=0.9 \mathrm{~mm})$ and can be calculated by the equation in [17]. The equivalent metallic waveguide model is used to speed up calculation in a full-wave simulation software. The width of the radiating slots $w_{\mathrm{s}}$ is chosen as $0.5 \mathrm{~mm}$ which satisfies the narrow slot assumption in consideration of the operating bandwidth and fabrication tolerances [18]. The width of the SIW, $a=15.0 \mathrm{~mm}$, is chosen as equal to half the free-space wavelength $\left(\lambda_{0} / 2\right)$ at the center frequency of $10.0 \mathrm{GHz}$ to avoid grating lobes when steering beams to large angles. The beam is steered by exciting the array with equal amplitudes and progressive phase shifts in the E-plane (xoz-plane). The properties of the substrate filled in the SIW are chosen as $\varepsilon_{\mathrm{r} 1}=2.2$ and $t_{1}=1.43 \mathrm{~mm}$.

The parameters of all radiating slots, including lengths and offsets, of the SIW array are synthesized by using the standard Elliott's iterative procedure. A complete SIW slot array is modeled and simulated in full-wave simulation software CST. The first radiating slot of each waveguide is set as the same offset. The image effect of the last slot of each radiating guide is compensated.

\section{ELC Resonator}

An ELC resonator has a strong electric response, and it is suitable for implementing media with desired negative permittivity [16]. It also can be used as a transmissive structure 


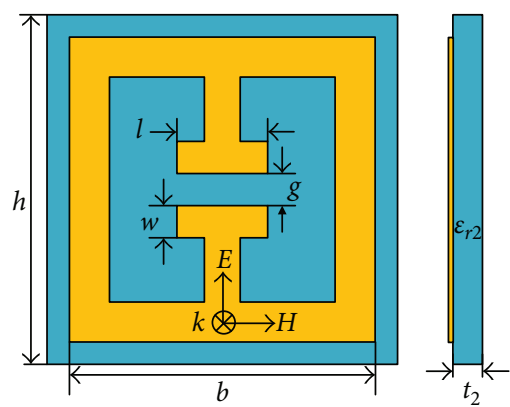

(a)

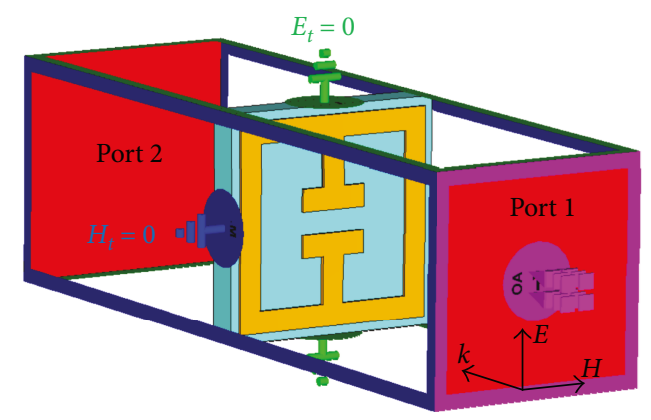

(b)

FIgURE 2: (a) Configuration of the square ELC resonator and (b) boundary conditions and polarization of an incident wave.

for the electromagnetic wave to change some components of the E-field. Figure 2(a) shows the conventional ELC resonator unit which can be used for WAIM slabs. The ELC resonator structure is supported by a substrate with a relative permittivity of $\varepsilon_{\mathrm{r} 2}=2.2$ and a thickness of $t_{2}=0.43 \mathrm{~mm}$, and the thickness of the coating metal is $0.035 \mathrm{~mm}$. Here, we focus on studying the scattering properties of the metamaterial unit (especially the amplitude and phase of the transmission coefficient, S21). The ELC resonator unit is simulated in the CST Microwave Studio by employing proper electric and magnetic boundary conditions on the transverse boundaries and two open ports (see Figure 2(b)) to obtain the $S$-parameter response of a single infinite layer medium to a normally incident plane wave.

As shown in Figure 2(a), the plane wave propagates perpendicularly to the plane of the ELC resonator, with the electric field $E$, polarized perpendicularly to the gap. The simulated results for the scattering parameters are depicted in Figure 3. It shows the frequency response for two ELC resonators of different dimensions. It can be seen that the ELC unit of one-fifth wavelength, named as ELC2, forms a stop band around $10 \mathrm{GHz}$. The dimensions of the ELC2 resonator are optimized in CST to operate at the center frequency of $10 \mathrm{GHz}$ [16]. Then, all the structure parameters of the ELC unit are decreased to half except the thickness of the substrate $\left(t_{2}\right)$. The ELC resonator shifts its resonance point from $10 \mathrm{GHz}$ to $19 \mathrm{GHz}$. In this work, the ELC2 resonator is taken as a control group and the smaller ELC resonator is the research priority. The region of interest is highlighted in yellow at the X-band. In this region, the ELC unit leads a phase shift about $-47^{\circ}$, which delays more $41^{\circ}$ with respect to a vacuum unit with the same thickness as the ELC unit. Meanwhile, the amplitude of S21 is about $-2.6 \mathrm{~dB}$; that is to say, a little more than $50 \%$ power transmits through the ELC unit, only for TEM mode as shown in Figure 2. This characteristic is what we need to realize a WAIM structure in Section 4.

\section{Mutual Coupling Reduction Using the ELC Metasurface}

Using a metal slab between adjacent elements to reduce mutual coupling is a common way to improve scanning performance of the array. In fact, on the one hand, the mutual coupling from diffraction is considerable if the dimension of the metal slab is limited. On the other hand, the performance of wide-angle scanning is deteriorated if the metal slab is too large. So some researchers proposed a metamaterial slab operating at epsilon-negative band to realize decoupling [13]. This idea corresponds to the ELC2 resonator proposed in Section 3, but it shows the characteristics resembling a metal slab. The metal slab and ELC2 metasurface slab are both control groups to be compared with the ELC metasurface slab in this section.

The SRR metasurface slab, which lies above an antenna and possesses a wave-transmissive property, requires a magnetic vector normal to its plane so that it can generate a response (i.e., a wave with a TE-polarized component with respect to the sheet's plane). The SRRs are excited and offer increasing compensation in $D$ - and $H$-planes, but they are effectively invisible in the E-plane as the beam is scanned from the broadside to the horizon [2]. Based on this view, an ELC metasurface slab of a transmissivity, standing on the SIW slot array, can generate a response for an electric vector parallel to its plane (i.e., a TE- or TEM-polarized wave with respect to the slab's plane). The designed ELC metasurface is a semipermeable structure for the electromagnetic wave. Most coupling field will penetrate the ELC slabs of $\lambda_{0} / 10$ height. Then, the vertical component of the penetrating E-field will be decreased, which leads to the reduction of mutual coupling between two adjacent SIWs. As a result, the ELC metasurface can mitigate the impedance mismatch, which is mainly caused by mutual coupling from the adjacent radiating waveguide, when the array is excited by progressive phase shift as the beam is scanned off the broadside.

We have studied the mutual coupling in the four cases: (1) without WAIM, (2) with an ELC2 metasurface slab of $4 \lambda_{0} / 10$ height, (3) with a metal slab of $4 \lambda_{0} / 10$ height, and (4) with an ELC metasurface slab of $4 \lambda_{0} / 10$ height. The SIW slot array in the four cases is built and simulated, respectively, in CST MWS using a time-domain solver. Figure 4 shows the electric field vector distribution between two waveguides when port 5 is excited in the four cases. From Figure 4(a), it can be seen that the coupling energy from the left slot to the right slot is the strongest of the four cases, which leads to a serious impedance mismatching when the phase difference of the excitation between port 5 and port 6 increases (i.e., when the scan beam is steered 


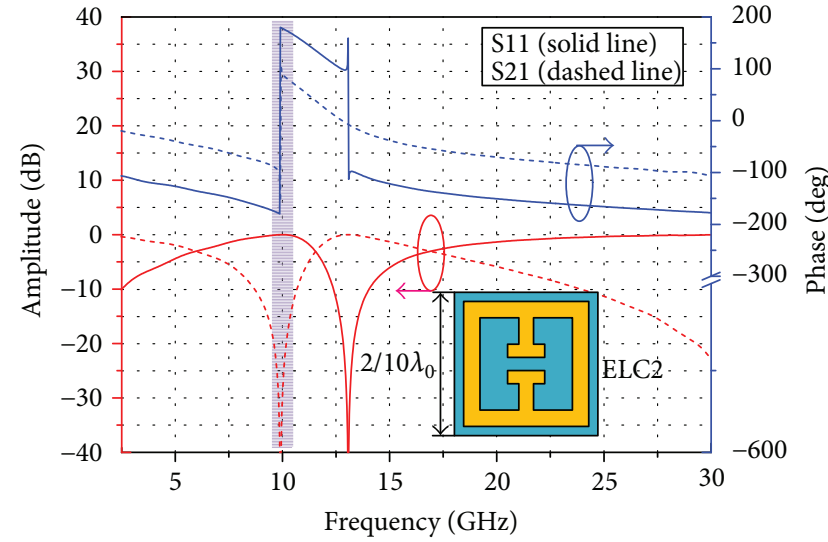

(a)

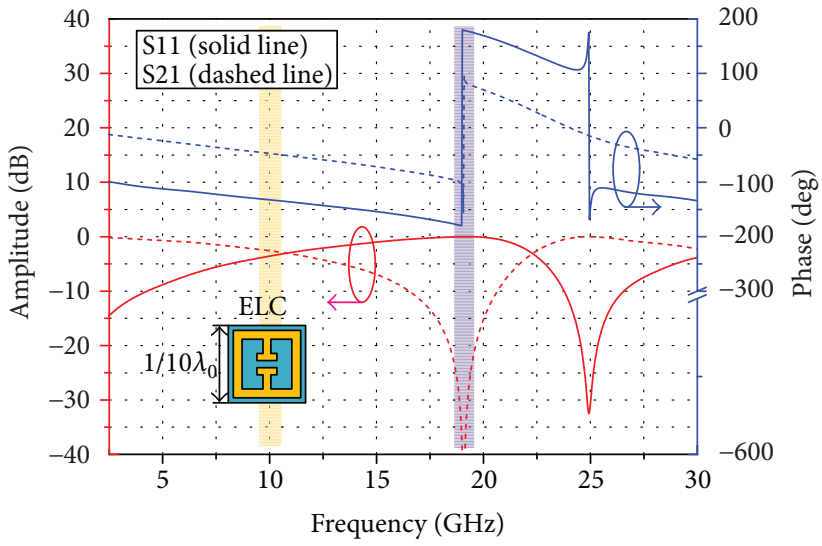

(b)

Figure 3: (a) S-parameters of the ELC2 resonator: $b=5.52 \mathrm{~mm}, h=6.0 \mathrm{~mm}, g=0.6 \mathrm{~mm}, l=1.8 \mathrm{~mm}, t_{\mathrm{s}}=0.43 \mathrm{~mm}$, and $w=0.6 \mathrm{~mm}$. (b) $S$-parameters of the ELC resonator: $b=2.76 \mathrm{~mm}, h=3.0 \mathrm{~mm}, g=0.3 \mathrm{~mm}, l=0.9 \mathrm{~mm}, t_{\mathrm{s}}=0.43 \mathrm{~mm}$, and $w=0.3 \mathrm{~mm}$ (the operating band in this work is located in the yellow zone, the electric field response shows insulation property in the purple zone, and its corresponding permittivity is negative).

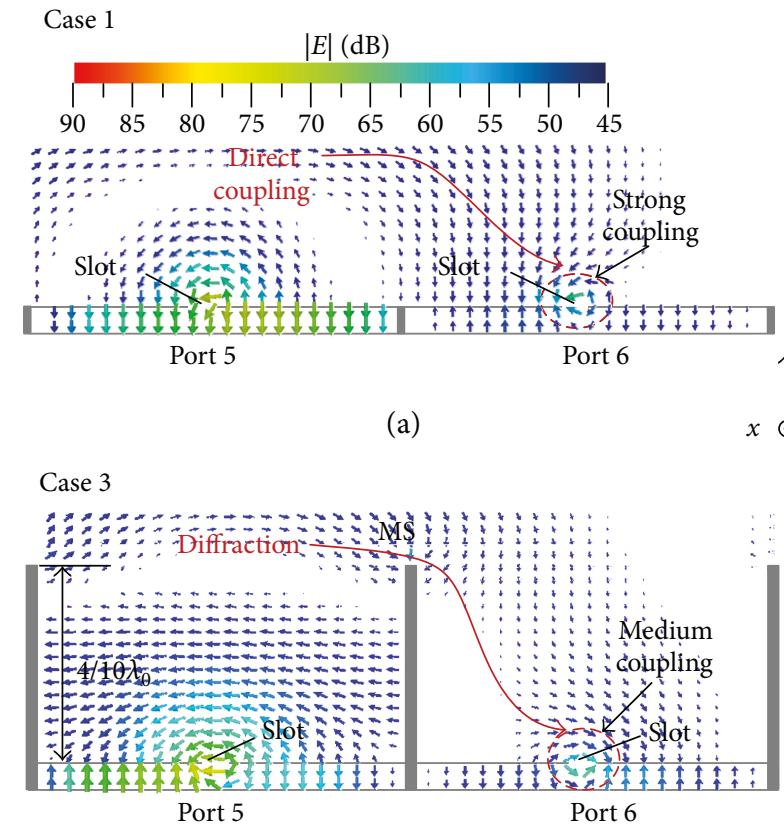

(c)

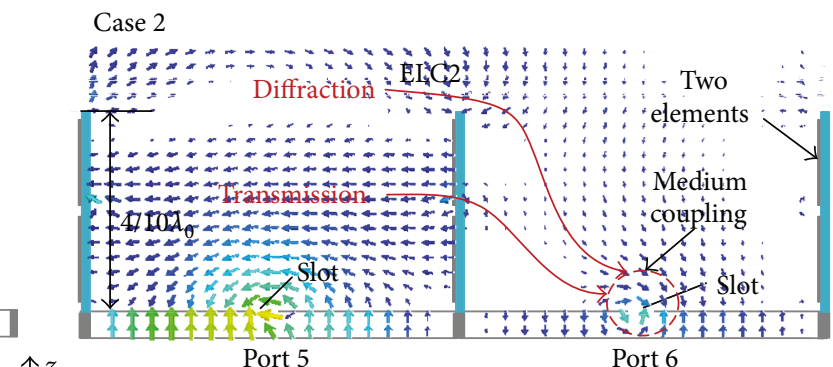

(b)

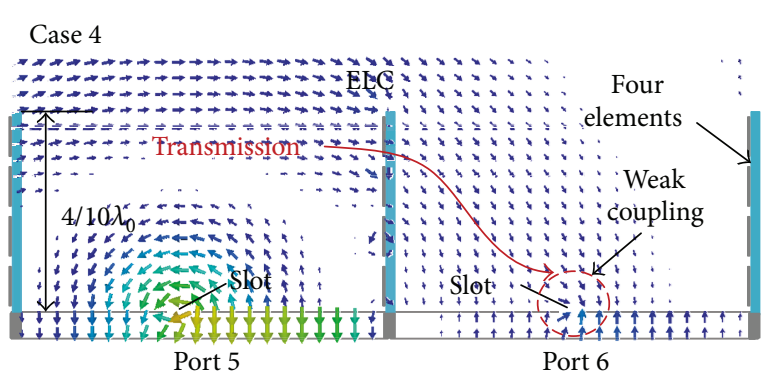

(d)

FIGURE 4: Cross-sectional E-field mutual coupling between two adjacent SIWs at $10.0 \mathrm{GHz}$ in four cases: (a) case 1: without WAIM, (b) case 2: with the ELC2 metasurface slab, (c) case 3: with a metal slab (MS), and (d) case 4: with the ELC metasurface slab.

off the broadside). The energy from the left slot is mainly coupled to the right slot through the diffraction and slightly coupled by penetrating the slab in TM mode or other highorder modes as shown in Figure 4(b), in which the coupling amplitude is weakened a little and its phase is delayed mainly depending on the path length. Figure 4(c) depicts that the energy from the left slot is completely coupled to the right slot along the diffraction path, which is almost the same with case 2. From Figures 4(b) and 4(c), it can be seen that the reduction in mutual coupling is not considerable with an ELC2 metasurface slab or a metal slab (less than $\lambda_{0} / 2$ height).
As seen in Figure 4(d), we find that the mutual coupling between the two radiating slots is reduced much more than those of the other three cases. This is attributed to the interesting properties of the ELC metasurface slab which can reduce the vertical ( $z$-direction) component of the electric field and change the polarization direction when the most part of the coupling wave penetrates the metasurface slab. As a result, the mutual coupling is greatly decreased.

Figure 5 indicates the mutual coupling between any two adjacent SIWs for the $10 \times 10$ slot array in the four cases. From Figure 5(a), it can be seen that metal slabs of $\lambda_{0} / 10$ to 


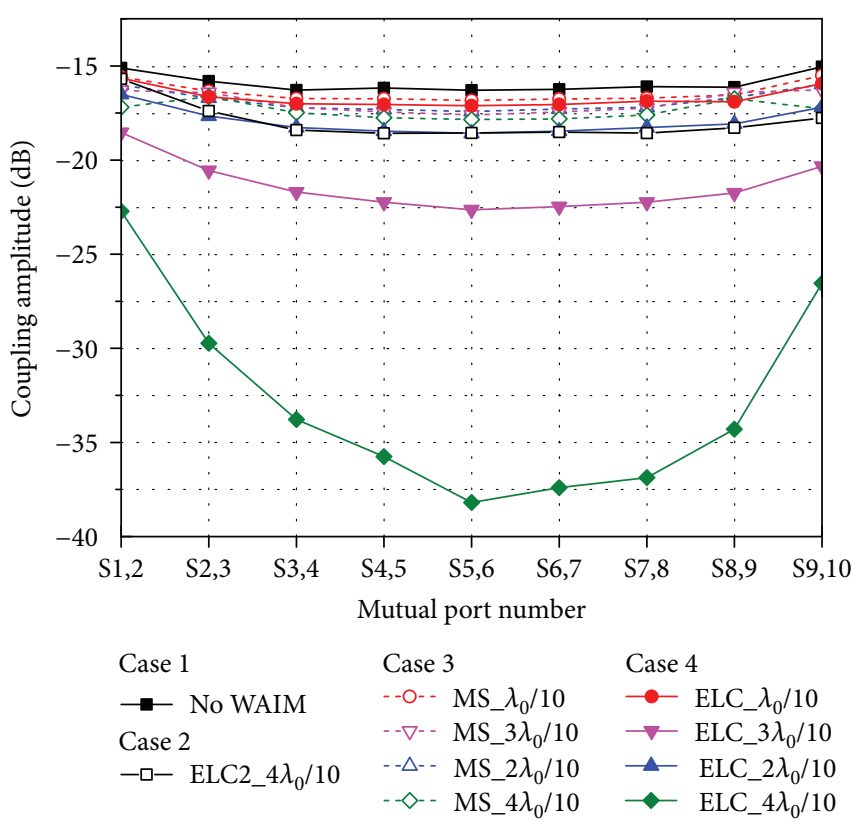

(a)

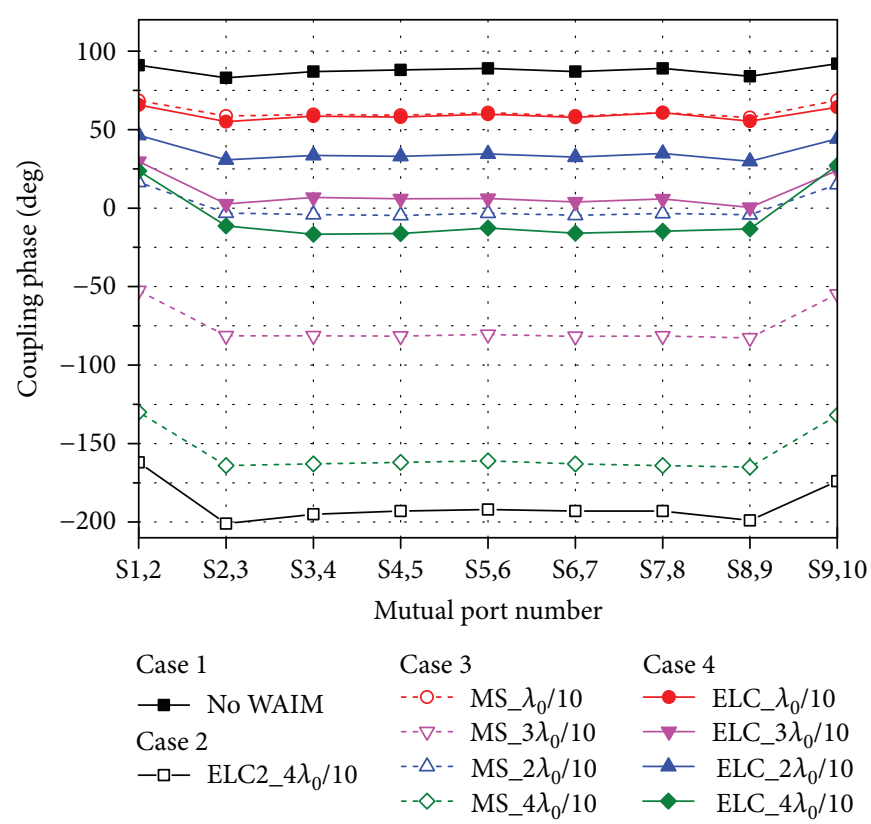

(b)

FIGURE 5: Mutual couplings between two adjacent SIWs at $10.0 \mathrm{GHz}$ in the four cases. (a) Amplitude and (b) phase. The value following the underline in the graph legends presents the height of the slab.

$4 \lambda_{0} / 10$ height and the ELC2 metasurface slab of $4 \lambda_{0} / 10$ just decrease the amplitude within $2 \mathrm{~dB}$ with respect to case 1 . However, ELC metasurface slabs of $\lambda_{0} / 10$ to $4 \lambda_{0} / 10$ height can cut down the amplitude from $-1 \mathrm{~dB}$ to $-32 \mathrm{~dB}$ with respect to case 1 , which have a remarkable improvement as the height increases. From Figure 3(b), we know that the ELC metasurface can cause phase delay for the transmitted wave in the linear region. For a slot array, the coupling fields are mainly concentrated within $\lambda_{0} / 2$ above the surface of the radiating array. It will lead to phase delay when the coupling wave penetrates the ELC slabs. From Figure 5(b), the phase delay in case 2 approximates the phase delay in case 3 with a slab height of $4 \lambda_{0} / 10$, and the tiny difference is caused by the transmission of a non-TE-polarized wave. The phase delay in case 3 increases with the diffraction path length which is determined by the height of the slab, while in case 4 , it has a limited change in a range of about $70^{\circ}$. The phase delay increases less and less as the height of the ELC slabs increases from $\lambda_{0} / 10$ to $4 \lambda_{0} / 10$. When the height of the ELC slabs exceeds $\lambda_{0} / 2$, the coupling phase will increase little. It concludes that the mutual coupling of the two adjacent radiating waveguides can be more reduced by ELC metasurface slabs than by metal slabs when the height is between $2 \lambda_{0} / 10$ and $4 \lambda_{0} / 10$.

The reflection coefficients for all input ports in the 4 cases are plotted in Figure 6(a). It can be seen that the ELC2 metasurface of $4 \lambda_{0} / 10$ height and the ELC metasurface of $\lambda_{0} / 10$ height make a worse matching, while other cases make a better matching for all input ports. In particular, the ELC metasurface of $3 \lambda_{0} / 10$ height doses better than the one of $4 \lambda_{0} / 10$ height except at port 1 and port 10 . Figure $6(\mathrm{~b})$ shows all the mutual couplings for port 1 . It can be seen that the mutual coupling for port 1 becomes smaller with the spacing distance between two ports increasing in the 4 cases. In particular, the mutual couplings (S14 S110) in case 4 are larger than those in the other cases. In cases $1 \sim 3$, most coupling power couples into the several adjacent waveguides. While in case 4, inductive currents on the ELC metasurface can generate inductive field one by one to couple into the waveguides far from the exciting waveguide. Even though, we could ignore the mutual couplings except the two adjacent waveguides because they have a little effect on the active impedance matching.

\section{Improvement of Wide-Scan Performance in the SIW Slot Array Antenna}

In order to validate the performance of wide-angle scanning, the width of an SIW is chosen as $a=15.0 \mathrm{~mm}$, which is the same as the array-element separation $(d x)$. The ideal maximum scan angle can be calculated by

$$
\frac{d x}{\lambda_{0}}=\frac{1}{1+\left|\sin \theta_{m}\right|} .
$$

By (1), $\theta_{m}$ is calculated as $90^{\circ}$ based on the $d x=15$ $\mathrm{mm}$ and $\lambda_{0}=30 \mathrm{~mm}$. However, this ideal maximum scan angle can be achieved under the condition that the element pattern is omnidirectional (i.e., the radiating element is a point source). In fact, the beamwidth of the slot element pattern limits the maximum scan angle of the slot array. That is to say, a grating lobe will appear at a scan angle of less than $90^{\circ}$. In this work, the actual maximum scan angle is about $72^{\circ}$.

The radiating efficiency and realized gains as functions of the scan angle for the SIW slot array without WAIM 


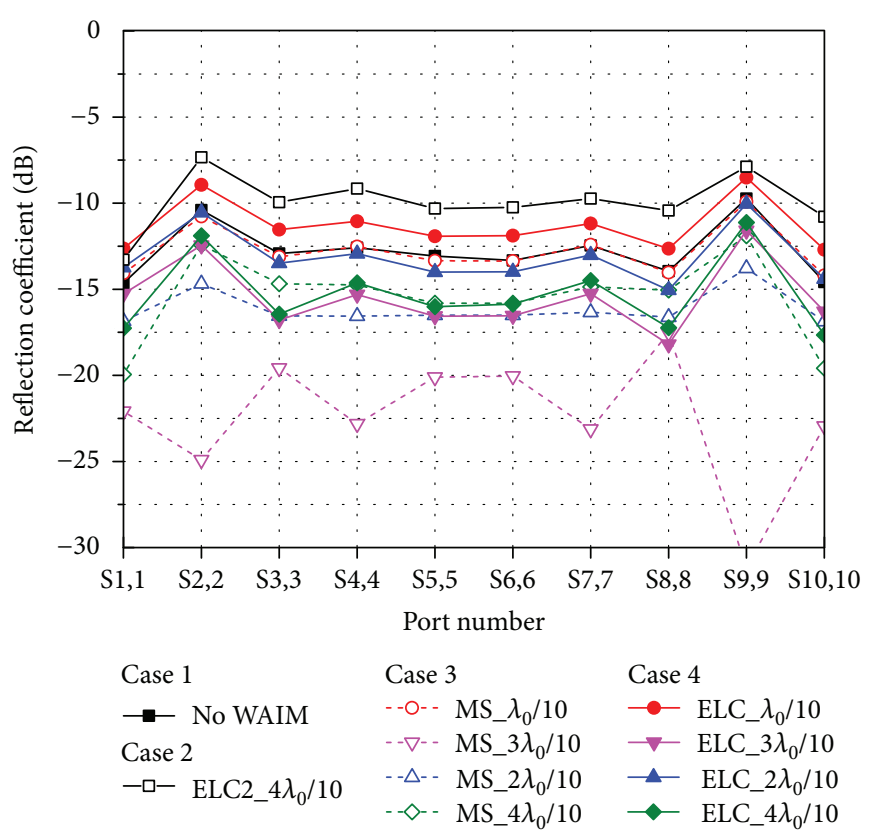

(a)

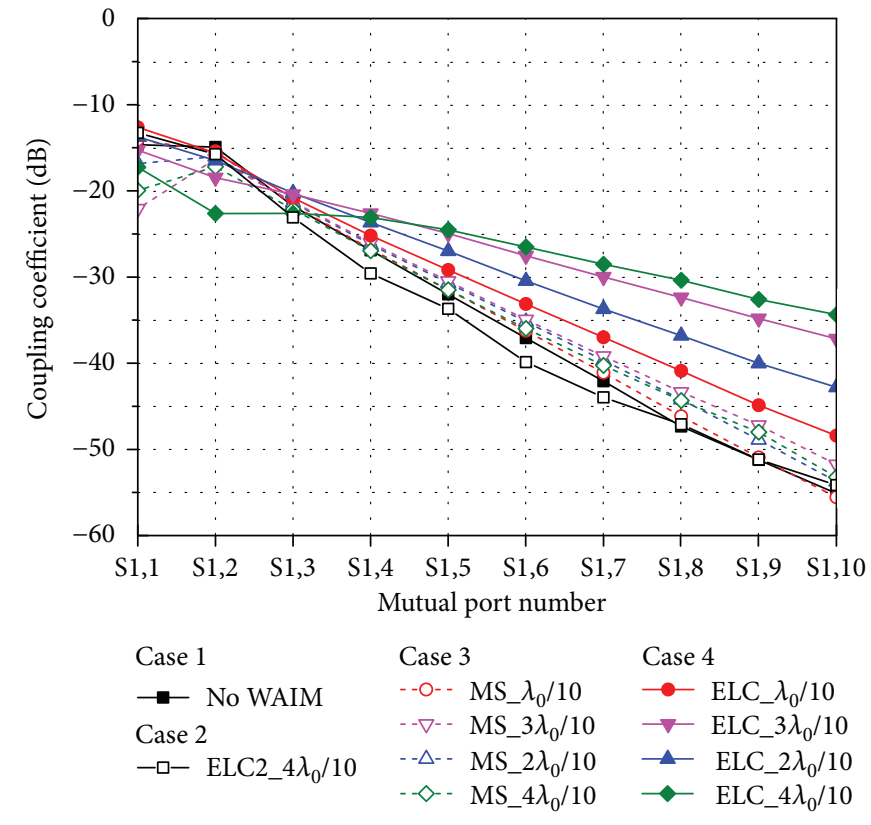

(b)

Figure 6: (a) Reflection coefficient for each port at $10.0 \mathrm{GHz}$ in the four cases. (b) Mutual couplings for port 1 at $10.0 \mathrm{GHz}$ in the four cases.

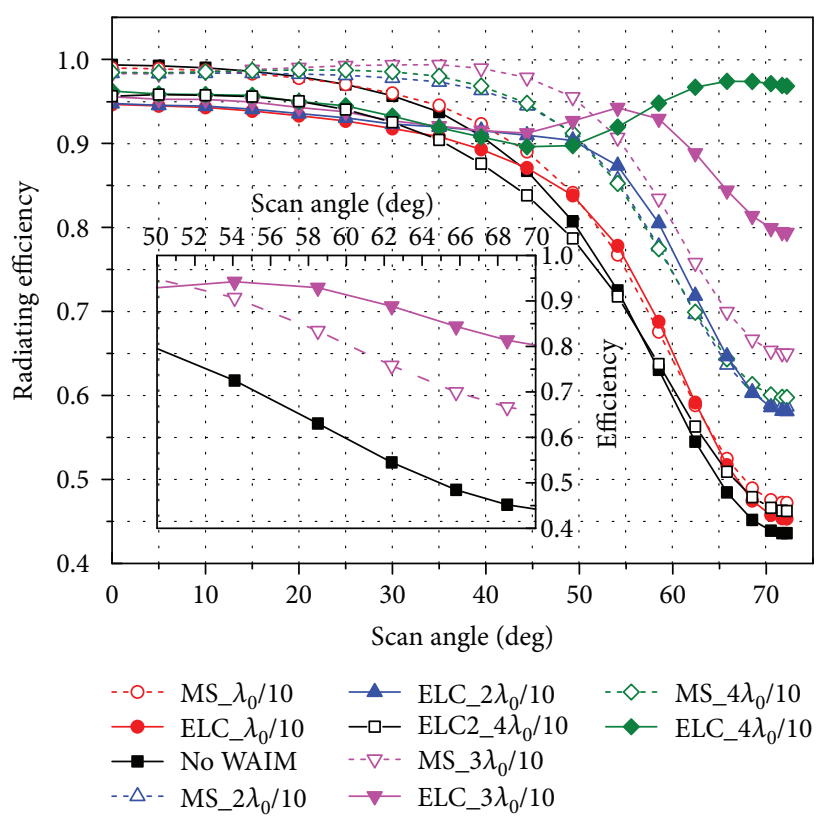

(a)

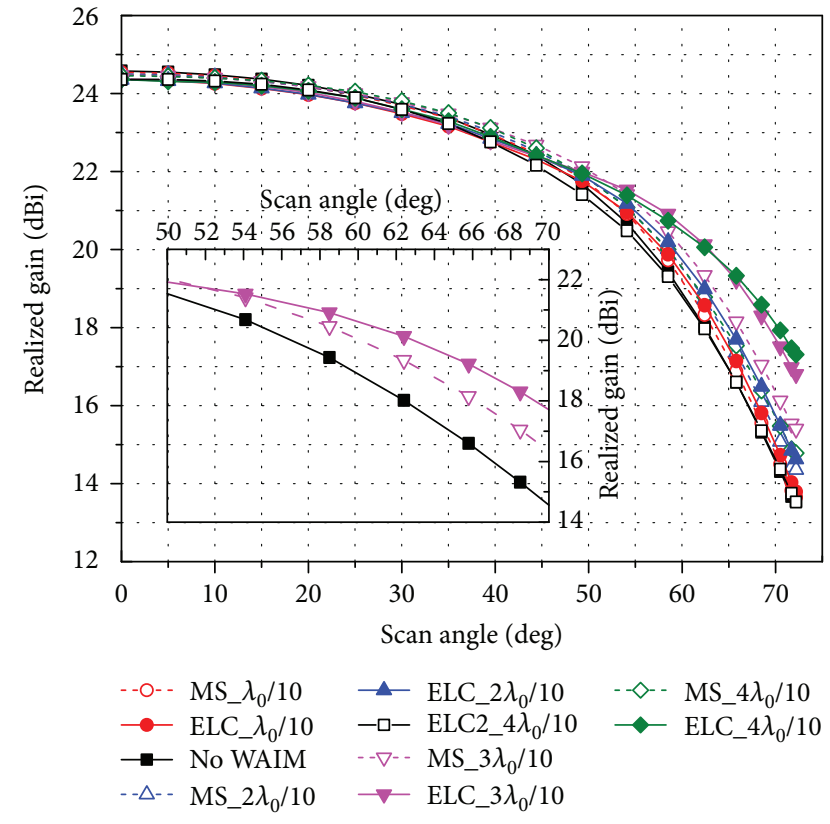

(b)

Figure 7: (a) Radiating efficiency and (b) realized gains as a function of the scan angle at $10.0 \mathrm{GHz}$ in the E-plane for the four cases.

and with the ELC2 metasurface slabs, the metal slabs, and the ELC metasurface slabs are plotted in Figure 7. From the efficiency point of view as shown in Figure 7(a), the ELC metasurface slab of $3 \lambda_{0} / 10$ height allows for a larger scan range in the $E$-plane than its metal slab counterpart of the same height, while the efficiency is little lower at a small scan angle and it adds an acceptable increase to the profile. The scan angle range, in which the efficiency is greater or equal to $80 \%$, is significantly improved from $50^{\circ}$ to $70^{\circ}$ when compared with the no-WAIM case. As a result, the ELC metasurface slab of $3 \lambda_{0} / 10$ height achieves a higher realized gain from $50^{\circ}$ to $70^{\circ}$ than case 1 as seen in Figure 7(b). The realized gain is a related quantity which uses the stimulated power instead of the accepted 
power as a reference quantity. This quantity considers the loss from the reflection and is defined by

$$
G_{\text {realized }}(\theta, \phi)=4 \pi \frac{P_{\mathrm{r}}}{P_{\mathrm{s}}}
$$

where $p_{\mathrm{r}}$ is the power radiated per unit solid angle and $P_{\mathrm{s}}$ is the stimulated power. It is worth noting that the efficiency drops off over the large scan angle range as the height of the metal slab increases from $3 \lambda_{0} / 10$ to $4 \lambda_{0} / 10$. This supports the view that a higher metal slab will block incident electromagnetic waves from low elevation angle.

So using an ELC metasurface slab of $3 \lambda_{0} / 10$ height to realize WAIM is a tradeoff between low scan loss and low profile. Figure 8 shows the scan beams from $0^{\circ}$ to $72^{\circ}$, comparing the ELC metasurface case with the no-WAIM case. In particular, the realized gain gets a $3.2 \mathrm{~dB}$ increase at a scan angel of $72^{\circ}$. Meanwhile, the grating lobes appearing at around $-72^{\circ}$ are within an acceptable level. It is clear that the ELC metasurface has a more significant impact on the usable range of the SIW slot array when compared to noWAIM and metal slab cases.

Since bandwidth is a critical figure of merit for a beam scanning antenna, it is important to consider the implications of the ELC metasurface slab on the bandwidth of the SIW slot array, which has a narrow relative bandwidth of about $4 \%(400 \mathrm{MHz})$ for a normal fixed beam. The frequency response of the metamaterial may change rapidly at frequencies close to the metamaterial particle resonance. For the ELC resonator shown in Figure 3(b), its resonance frequency is located at $19 \mathrm{GHz}$. Due to the large separation of the resonance frequency between $10 \mathrm{GHz}$ (operation frequency) and $19 \mathrm{GHz}$, the ELC resonator operates in a less frequency dispersive band which keeps away from the resonance frequency and has a little effect on the band of the slot array. Simulations have been run at the design frequency $(10 \mathrm{GHz})$ and $4 \%$ of the design frequency $(9.8$ to $10.2 \mathrm{GHz}$ ) to investigate the bandwidth of the slot array without WAIM and with ELC metasurface slabs. Compared results are shown in Figure 9. The ELC metasurface performs well at lower scan angles but suffers in large scan angles at $9.8 \mathrm{GHz}$. Overall, the radiating efficiency is improved across the scan range in the bandwidth.

Note that the ELC metasurface is scaled and tuned for the SIW slot arrays based on the view of the design presented in [12]. It has not been optimized, as this is a proof of concept in simulated validation for such structures. Indeed, optimization on the parameters of the ELC unit may could further improve the bandwidth, impedance matching, and realized gain over a wider scan angle. However, any parametric analysis for various design parameters of the ELC metasurface and even any trials for different metasurface units are left as a future work.

\section{Conclusion}

In this paper, a simple and low-cost modified method to implement wide-angle scanning for SIW slot array antennas has been proposed. This research provides a tradeoff strategy

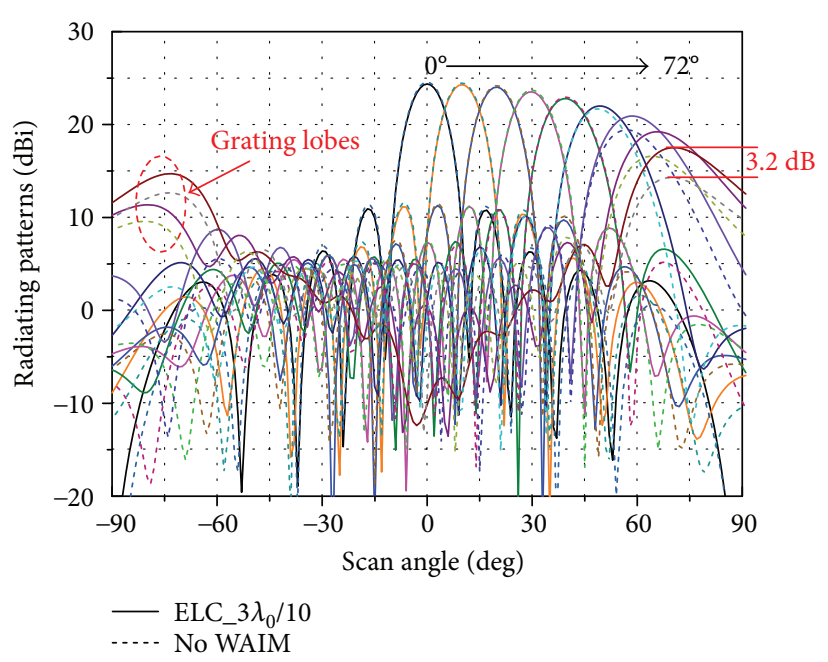

Figure 8: Simulated scan beams from $0^{\circ}$ to $72^{\circ}$ at $10.0 \mathrm{GHz}$ in two cases: without WAIM and with ELC metasurface slabs of $3 \lambda_{0} / 10$ height.

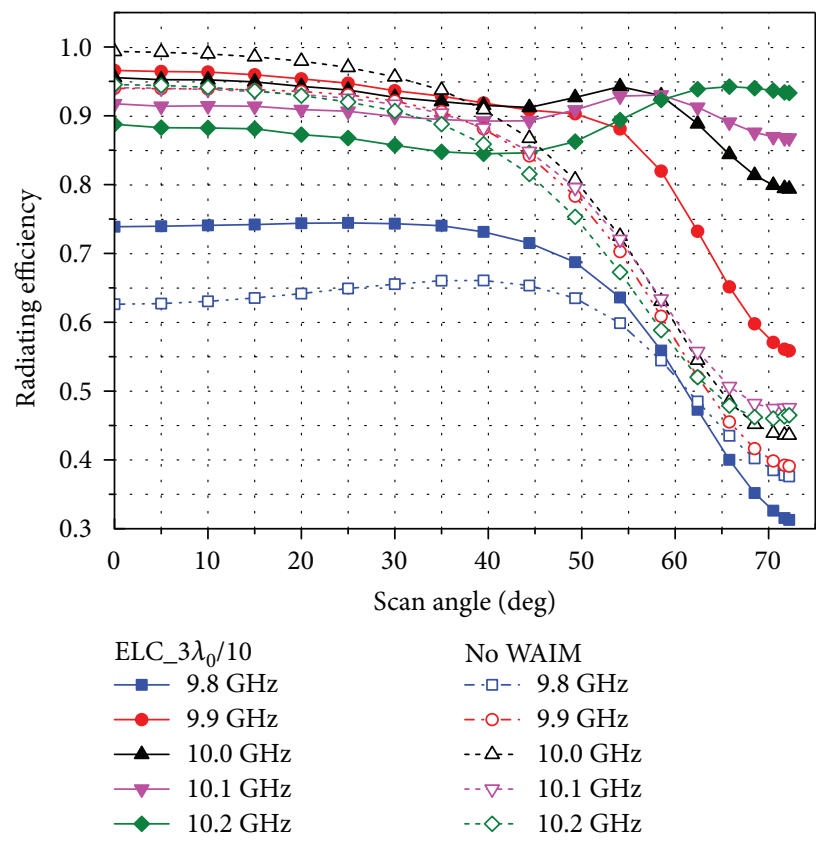

FIgURE 9: Simulated efficiency versus scan angle in the case without WAIM and with ELC metasurface slabs of $3 \lambda_{0} / 10$ height.

to improve the impedance matching in a wide range of scan angle. Using the transmissive characteristic of the ELC metasurface to realize WAIM has been qualitatively analyzed, and the WAIM in the E-plane has been remarkably improved for a $10 \times 10$ SIW slot array. The scanning properties of the SIW slot array with and without the ELC metasurface are numerically studied and compared. The simulated results indicate that the scan angle has been extended from $-50^{\circ}$ to $50^{\circ}$ to $-70^{\circ}$ to $70^{\circ}$. In conclusion, this approach can mitigate scan loss at large angles at little cost of gain loss at small angles. Structurally, the ELC metasurface adds around $\lambda_{0} / 4$ to the height of the antenna, maintaining a structure with an overall 
low profile in X-band applications. The ELC metasurface can be easily and quickly deployed in the waveguide slot arrays which require wide-angle scanning.

\section{Data Availability}

The data used to support the findings of this study are available from the corresponding author upon request.

\section{Conflicts of Interest}

The authors declare that they have no conflicts of interest.

\section{Acknowledgments}

This work is supported by the Major Applied Program for Key Basic Scientific Research of the National University of Defense Technology (ZDYYJCYJ20140701).

\section{References}

[1] N. Amitay, V. Galindo, and C. P. Wu, Theory and Analysis of Phased Array Antennas, John Wiley \& Sons, Chichester, UK, 1972.

[2] T. R. Cameron and G. V. Eleftheriades, "Experimental validation of a wideband metasurface for wide-angle scanning leaky-wave antennas," IEEE Transactions on Antennas and Propagation, vol. 65, no. 10, pp. 5245-5256, 2017.

[3] R. J. Mailloux, Phased Array Antenna Handbook, Artech House, Boston, MA, USA, 2005.

[4] R. Wang, B.-Z. Wang, X. Ding, and X.-S. Yang, "Planar phased array with wide-angle scanning performance based on image theory," IEEE Transactions on Antennas and Propagation, vol. 63, no. 9, pp. 3908-3917, 2015.

[5] G. Yang, J. Li, D. Wei, and R. Xu, "Study on wide-angle scanning linear phased array antenna," IEEE Transactions on Antennas and Propagation, vol. 66, no. 1, pp. 450-455, 2018.

[6] Y.-F. Cheng, X. Ding, W. Shao, M.-X. Yu, and B.-Z. Wang, "2-D planar wide-angle scanning-phased array based on wide-beam elements," IEEE Antennas and Wireless Propagation Letters, vol. 16, pp. 876-879, 2017.

[7] G. Yang, J. Li, S. G. Zhou, and Y. Qi, “A wide-angle E-plane scanning linear array antenna with wide beam elements," IEEE Antennas and Wireless Propagation Letters, vol. 16, pp. 29232926, 2017.

[8] Y.-Q. Wen, B.-Z. Wang, and X. Ding, "A wide-angle scanning and low sidelobe level microstrip phased array based on genetic algorithm optimization," IEEE Transactions on Antennas and Propagation, vol. 64, no. 2, pp. 805-810, 2016.

[9] C.-M. Liu, S.-Q. Xiao, H. L. Tu, and Z. Ding, "Wide-angle scanning low profile phased array antenna based on a novel magnetic dipole," IEEE Transactions on Antennas and Propagation, vol. 65, no. 3, pp. 1151-1162, 2017.

[10] E. Magill and H. Wheeler, "Wide-angle impedance matching of a planar array antenna by a dielectric sheet," in 1965 Antennas and Propagation Society International Symposium, pp. 164-169, Washington, DC, USA, 1965.

[11] S. Sajuyigbe, M. Ross, P. Geren, S. A. Cummer, M. H. Tanielian, and D. R. Smith, "Wide angle impedance matching metamaterials for waveguide-fed phased-array antennas,"
IET Microwaves, Antennas \& Propagation, vol. 4, no. 8, p. $1063,2010$.

[12] T. R. Cameron and G. V. Eleftheriades, "Analysis and characterization of a wide-angle impedance matching metasurface for dipole phased arrays," IEEE Transactions on Antennas and Propagation, vol. 63, no. 9, pp. 3928-3938, 2015.

[13] M.-C. Tang, S. Xiao, B. Wang, J. Guan, and T. Deng, "Improved performance of a microstrip phased array using broadband and ultra-low-loss metamaterial slabs," IEEE Antennas and Propagation Magazine, vol. 53, no. 6, pp. 3141, 2011.

[14] Z. Jin, G. Montisci, G. A. Casula, H. Yang, and J. Lu, "Efficient evaluation of the external mutual coupling in dielectriccovered waveguide slot arrays," International Journal of Antennas and Propagation, vol. 2012, Article ID 491242, 7 pages, 2012.

[15] Y. Liu, H. Yang, Z. Jin, F. Zhao, and J. Zhu, "Compact Rotman lens-fed slot array antenna with low sidelobes," IET Microwaves, Antennas \& Propagation, vol. 12, no. 5, pp. 656661, 2018.

[16] D. Schurig, J. J. Mock, and D. R. Smith, "Electric-field-coupled resonators for negative permittivity metamaterials," Applied Physics Letters, vol. 88, no. 4, article 041109, 2006.

[17] F. Xu and K. Wu, "Guided-wave and leakage characteristics of substrate integrated waveguide," IEEE Transactions on Microwave Theory and Techniques, vol. 53, no. 1, pp. 66-73, 2005.

[18] G. Montisci, Z. Jin, M. Li et al., "Design of multilayer dielectric cover to enhance gain and efficiency of slot arrays," International Journal of Antennas and Propagation, vol. 2013, Article ID 917676, 6 pages, 2013. 


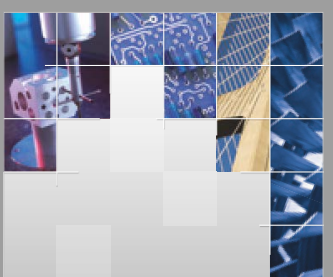

\section{Enfincering}
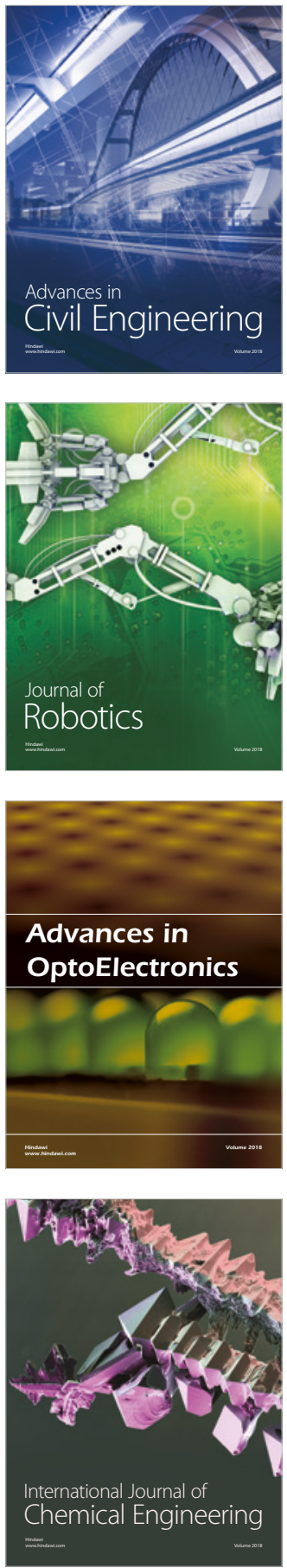

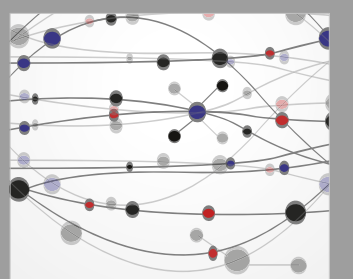

\section{Rotating \\ Machinery}

The Scientific World Journal

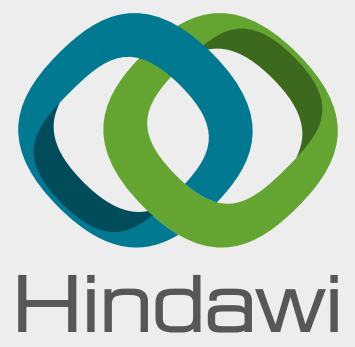

Submit your manuscripts at

www.hindawi.com
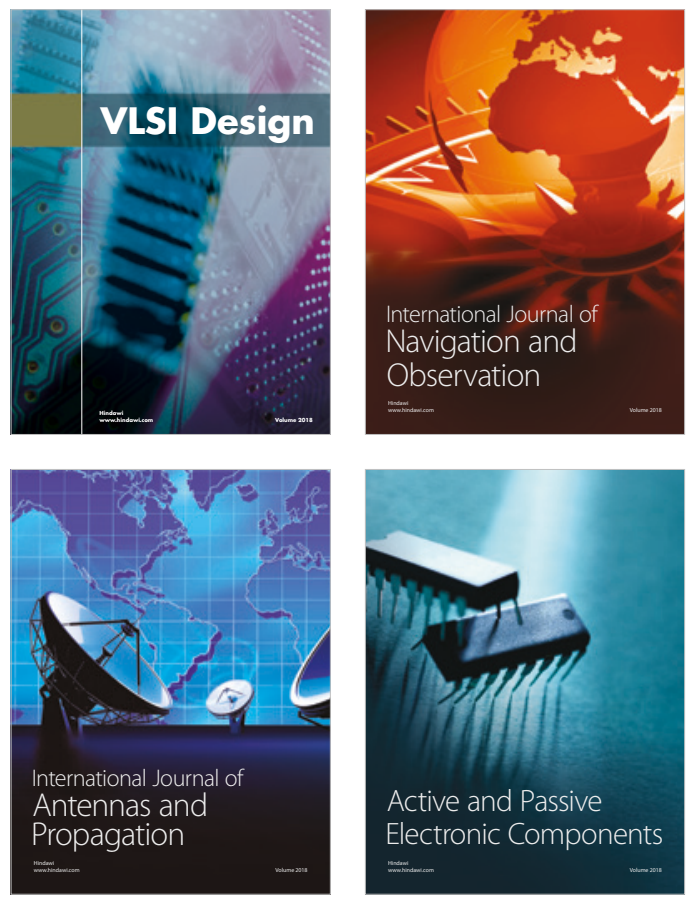
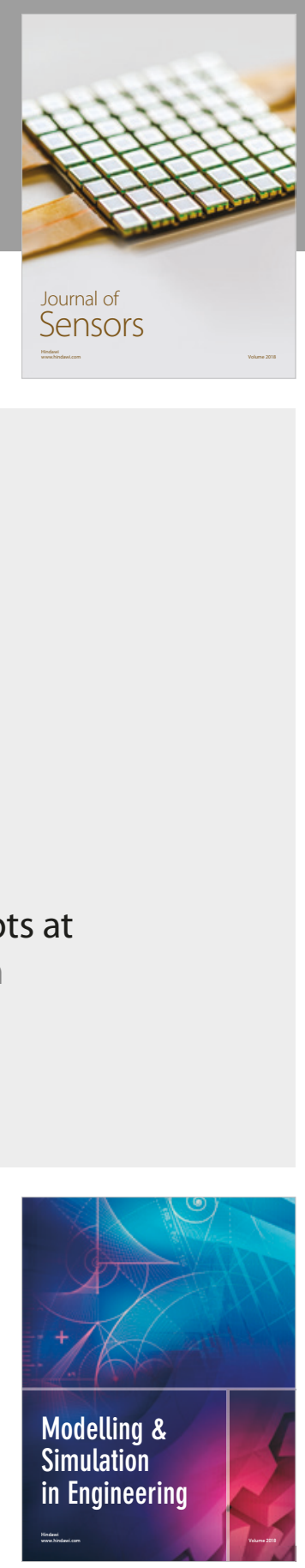

\section{Advances \\ Multimedia}
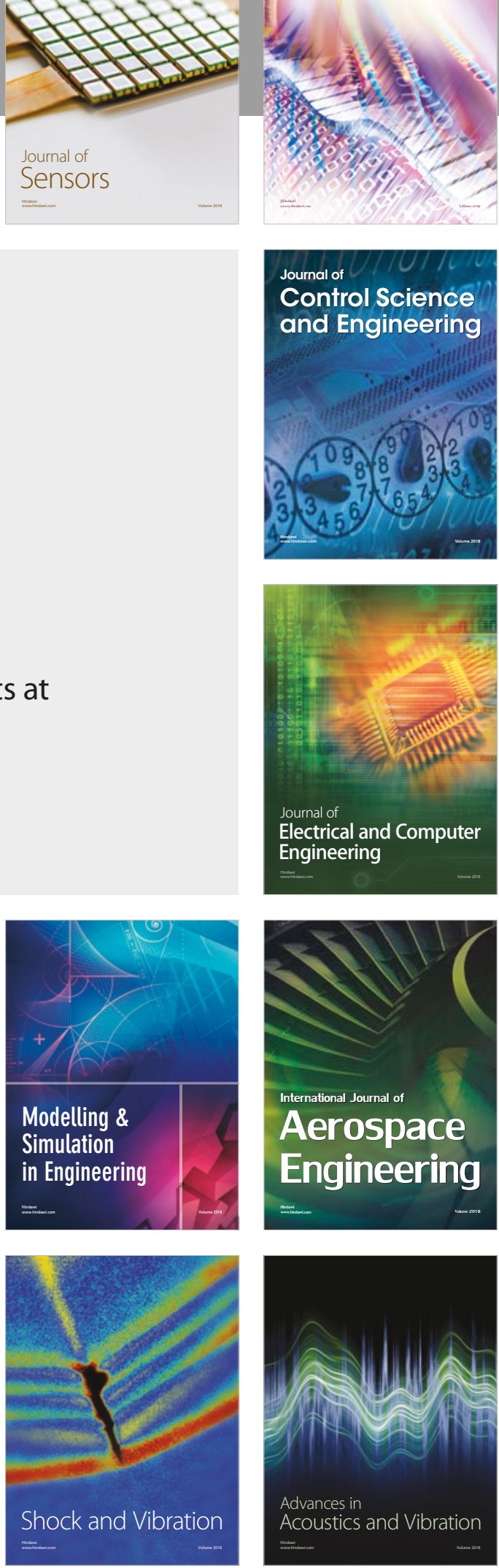